\title{
ISHAM \\ Ras-MAPK pathway participates of the nitrosative stress response in pathogenic fungus Paracoccidioides brasiliensis
}

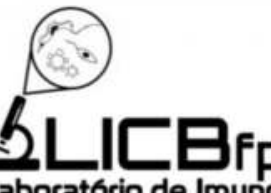 \\ Laboratorio de Imunologia Celular e}

Correspondence: mendespalloma@gmail.com

\author{
Palloma Mendes Conceição? ${ }^{2}$, Alison F. A. Chaves ${ }^{1}$, Daniele G. Castilho ${ }^{1}$, Marina V. Navarro; Juliana
} C. P. Calado ${ }^{1}$, Patricia Xander², Wagner L. Batista ${ }^{1,2}$

1 Department of Microbiology, Immunology and Parasitology/UNIFESP - São Paulo, Brazil

${ }^{2}$ Department of Pharmaceutical Sciences/UNIFESP - Diadema, Brazil.

\section{INTRODUCTION}

Paracoccidioides brasiliensis is a temperature-dependent dimorphic fungus that cause paracoccidioidomycosis (PCM). The capacity to evade the innate immune response of the host is due to its ability to respond and to survive the nitrosative stress caused by cells of the immune system. However, the regulation of signal transduction pathways associated to nitrosative stress response are poorly understood. Ras GTPases (Ras1 and Ras2) are well known to regulate antagonistically or cooperatively various cellular events in many fungi. Ras, in its activated form (Ras-GTP), interacts with effector proteins and can initiate a kinase cascade. In lower eukaryotes, Byr2 kinase represents a Ras target. In present study, we investigated the role of Ras GTPase in P. brasiliensis after in vitro stimulus with nitric oxide (NO). We observed that low concentrations of NO induce cell proliferation in P. brasiliensis, while high concentrations promoted decrease in fungal viability. Additionally, we observe that both events (cell proliferation and death) are reversed in the presence of a NO scavenger (carboxy-PTIO). We reported by immunobloting that after stimulation with NO, the Ras active form was observed in fungal extracts. High NO concentrations induces the $S$-nitrosylation of Ras in $P$. brasiliensis and, in this conditions, Ras modulates the expression of antioxidant genes in response to nitrosative stress. We also investigated whether Hog-1 would be activated in response to nitrosative stress. Under these conditions we observed that Hog-1 is phosphorylated after NO treatment. In addition, when we used the RBD-GST probe and blotted with anti-Hog-1 antibody we observed that Ras-GTP can interact with MAPK Hog-1 after stimulation with high concentrations of NO. Finally, our data indicate that Ras GTPase and Hog-1 pathways may cross-talk in response to nitrosative stress in P. brasiliensis. S-nitrosylation of Ras probably activates this GTPase, which initiates a signaling cascade involving Ras-Byr2-By1-Hog. These responses could help the fungus to survive after their initial contacts with the host immune system, which is crucial for disease establishment.

\section{MATERIALS AND METHODS}

We constructed an expression plasmid containing the Byr2 Ras-binding domain (RBD) fused with GST (RBD-GST probe, which detects the Ras active form). Immunobloting assays were performed to detect the Ras and Hog-1 activation. Ras and Hog-1 interaction were performed by immunoprecipitation assays.

\section{RESULTS}

A

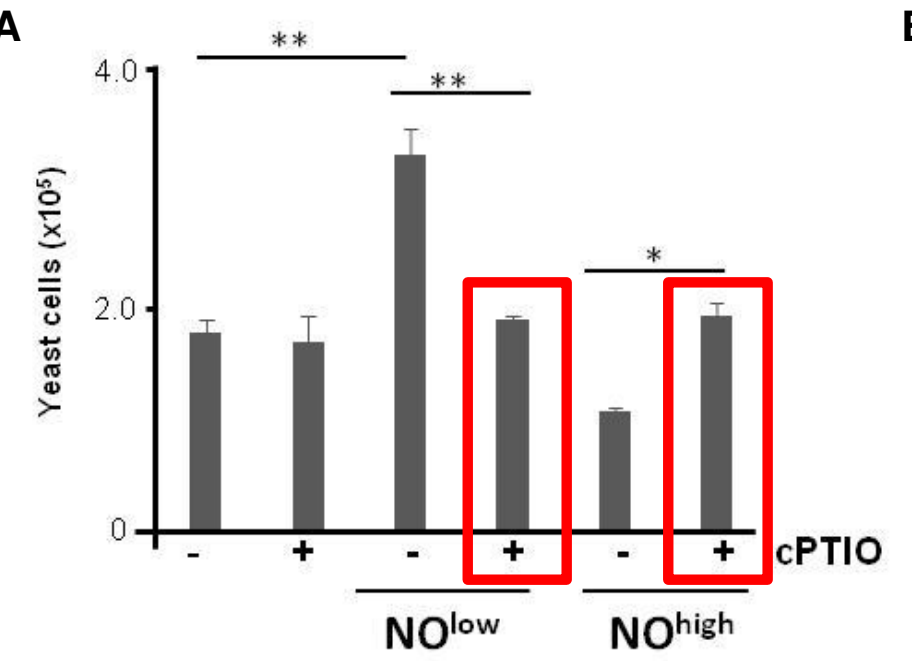

B

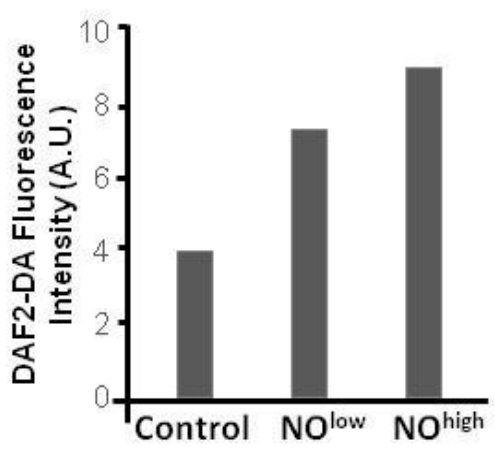

Figure 1: NO promoted cell proliferation in $\boldsymbol{P}$. brasiliensis. (A) At low concentrations of NO the fungus proliferated and developed better when compared to control. At high concentrations we observed decrease in fungal viability (as expected). (B) We also showed that these NO donors were in fact producing NO within the yeast. And finally, we observe that these events (both cell proliferation and death) are reversed in the presence of an NO scavenger (CPTIO).

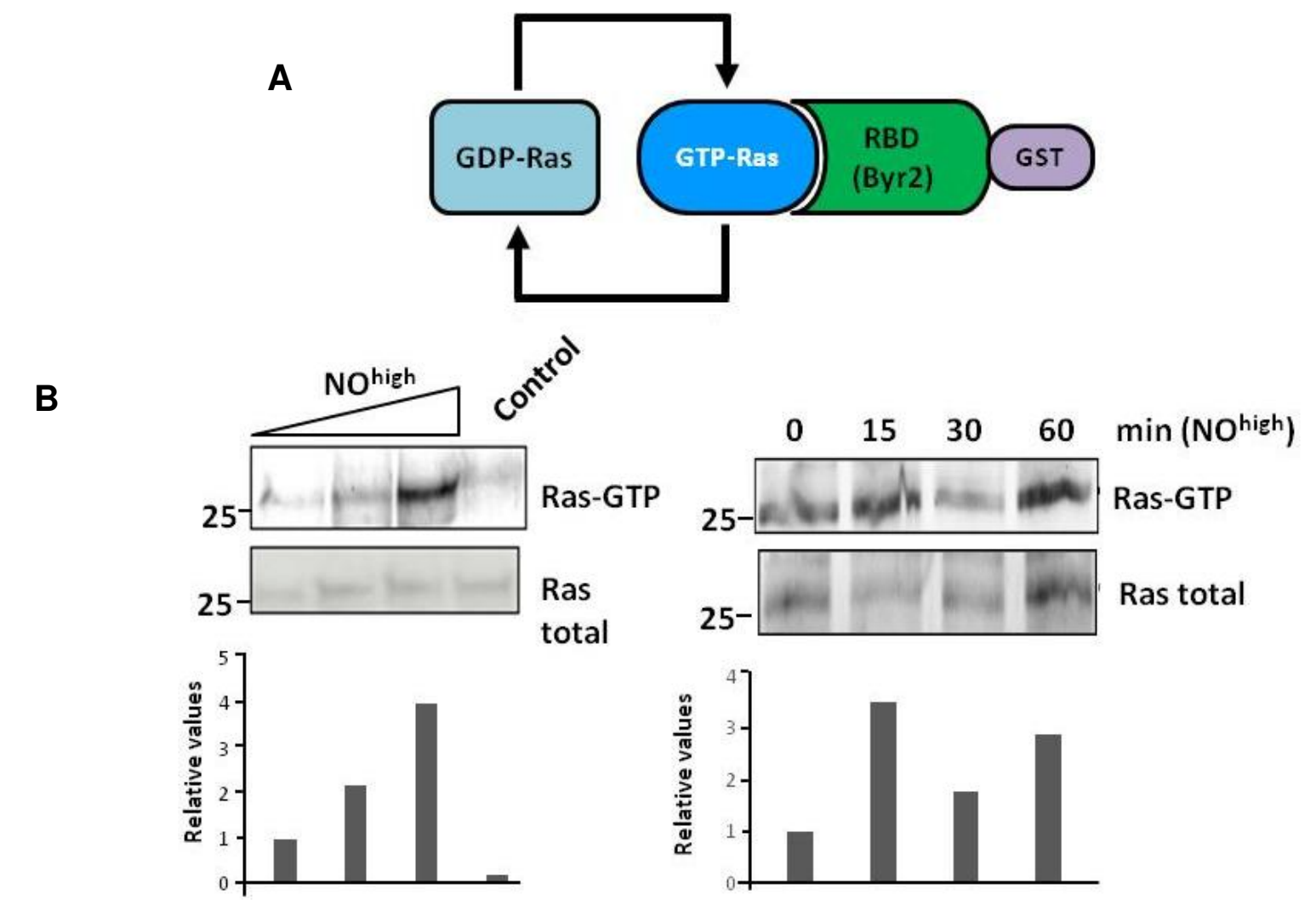

Figure 2: High concentrations of NO induces Ras activation in P. brasiliensis. (A) Ras is a GTPase that cycles between its form bound to GTP (active form) and the form bound to GDP (inactive form). Thus, we constructed a probe that detects Ras active form (Ras-GTP). In fungi, Byr2 (a the serine/threonine kinase) interact with activated Ras. The region Ras Binding Domain (RBD) was fused with GST. (B) Higher NO concentrations induced strong Ras activation. Suggesting that Ras participate of response to nitrosative stress.

A

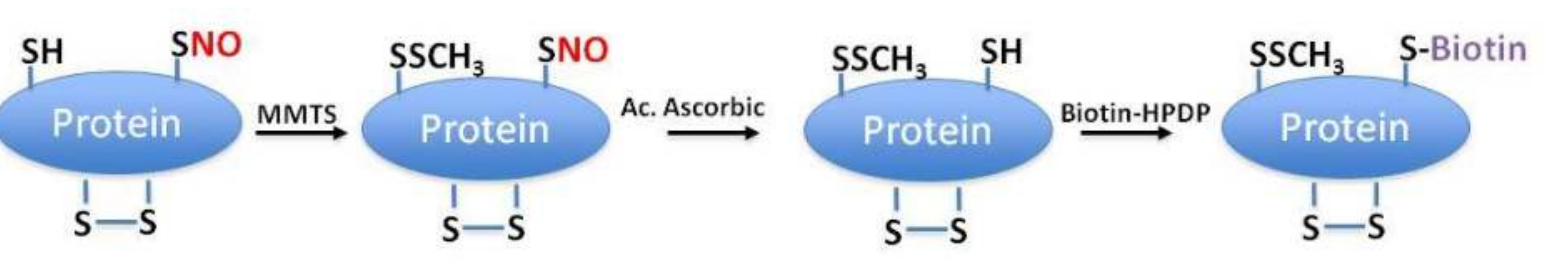

Figure 3: High concentrations of NO induces the Snitrosylation of Ras in $\boldsymbol{P}$. brasiliensis. We evaluated whether Ras would also be nitrosylated under high concentrations of NO. After analysis by the Biotin Switch Method assay (A), we found that high concentrations of NO induced rapid increase in S-nitrosylated Ras levels. These findings suggested that NO probably plays an important role in the process of Ras activation through its $S$-nitrosylation (B)

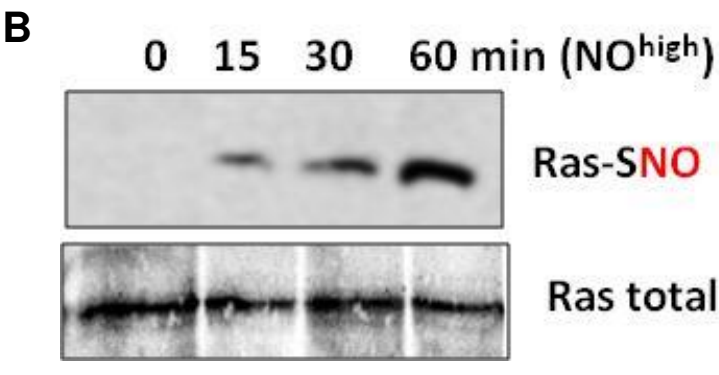

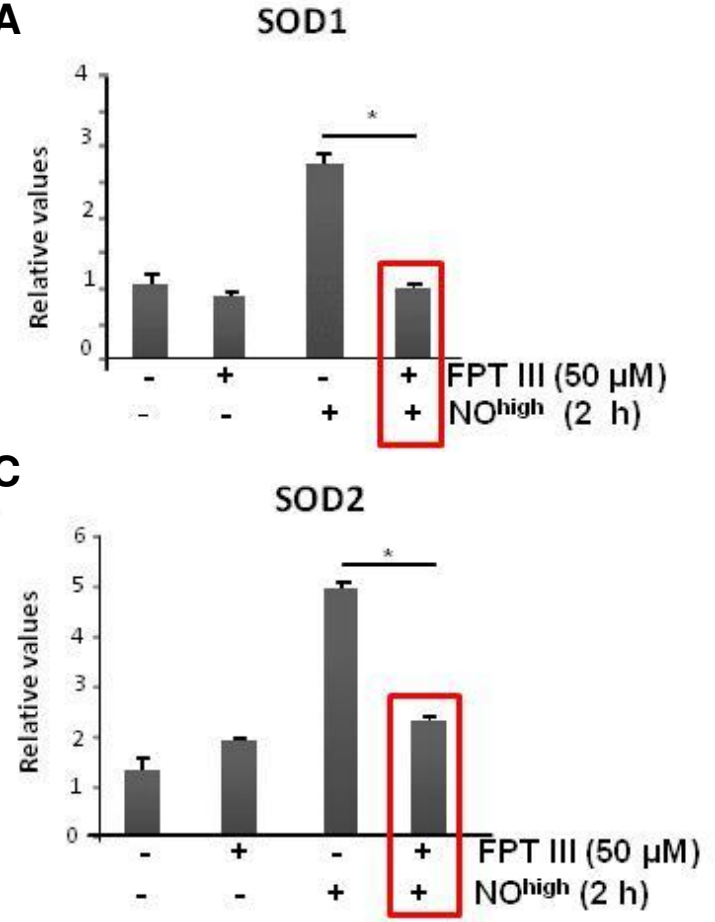

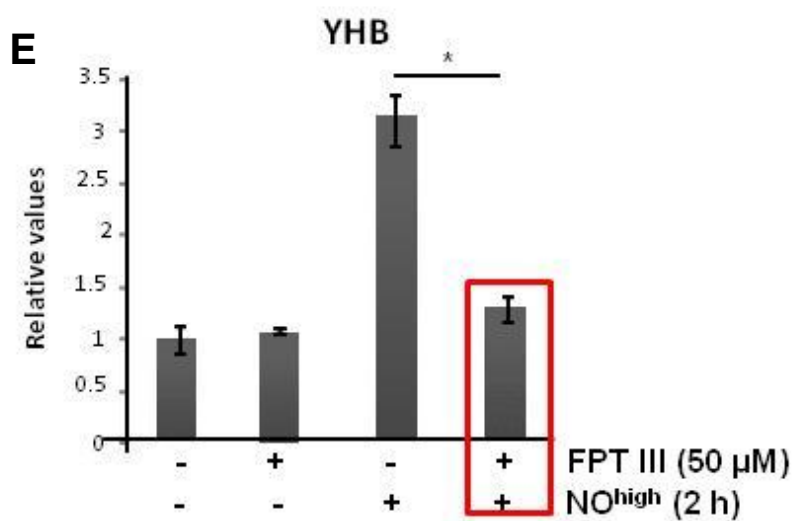

GR

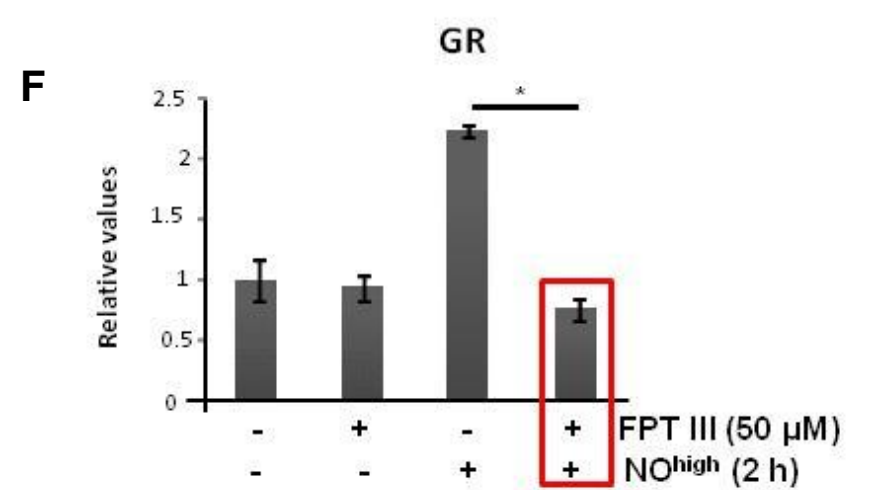

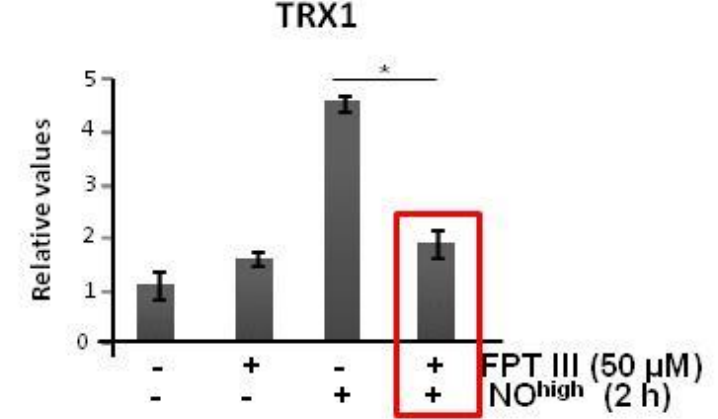

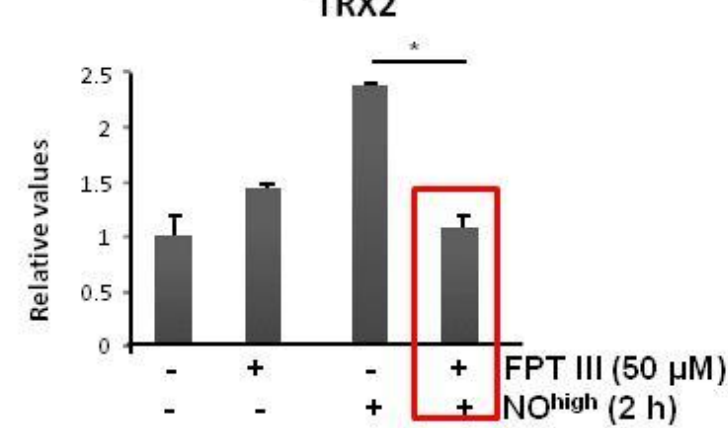

Figure 4: Ras modulates the expression of antioxidant genes in response to nitrosative stress. Due the Ras activation in response to high concentrations of NO, we evaluated by RT-qPCR the expression of genes related to the response to oxidative and nitrosative stress. For this, was performed in the presence and absence of the Ras inhibitor (FPT III). In this figure we can observe that the genes (A) SOD1 (superoxide dismutase 1), (B) TRX1 (Thioredoxin 1), (C) SOD2 (superoxide dismutase 2), (D) TRX2 (Thioredoxin 2), (E) YHB (important gene involved in the response to nitrosative stress) and (F) GR glutathione reductase) had their expression inhibited after treatment with the Ras inhibitor.
A

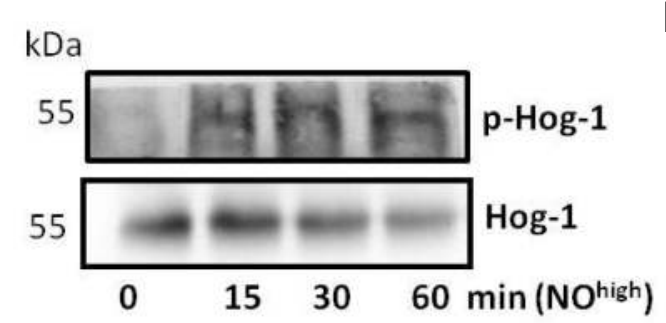

B

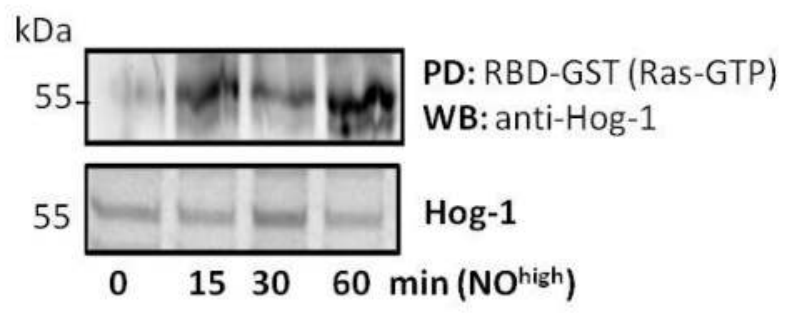

Figure 5: Ras is involved in the Hog1 pathway in response to nitrosative stress. Recently kinase Hog-1 has been shown to participate in the response to oxidative stress. Thus, we investigated whether Hog-1 would be activated in response to nitrosative stress. Under these conditions we observed that (A) Hog-1 is phosphorylated after NO treatment. In addition, when we used the (B) RBD-GST probe (which detects the activated form of Ras) and blotted with anti-Hog1 antibody we observed that Ras-GTP can interact with Hog-1 after stimulation with high concentrations of NO.

\section{CONCLUSIONS}

NO can act as a signal transducer in $P$. brasiliensis

Depending on NO concentration, different events are modulated in P. brasiliensis; High concentrations of NO can switch the Ras-GDP to Ras-GTP with consequent activation and triggering a response to nitrosative stress.

Ras is involved in the Hog1 pathway required for nitrosative stress. 